\title{
Latent-Variable Synchronous CFGs for Hierarchical Translation
}

\author{
Avneesh Saluja and Chris Dyer \\ Carnegie Mellon University \\ Pittsburgh, PA, 15213, USA \\ \{avneesh, cdyer\}@cs.cmu . edu
}

\author{
Shay B. Cohen \\ University of Edinburgh \\ Edinburgh EH8 9AB, UK \\ scohendinf.ed.ac.uk
}

\begin{abstract}
Data-driven refinement of non-terminal categories has been demonstrated to be a reliable technique for improving monolingual parsing with PCFGs. In this paper, we extend these techniques to learn latent refinements of single-category synchronous grammars, so as to improve translation performance. We compare two estimators for this latent-variable model: one based on EM and the other is a spectral algorithm based on the method of moments. We evaluate their performance on a Chinese-English translation task. The results indicate that we can achieve significant gains over the baseline with both approaches, but in particular the momentsbased estimator is both faster and performs better than EM.
\end{abstract}

\section{Introduction}

Translation models based on synchronous contextfree grammars (SCFGs) treat the translation problem as a context-free parsing problem. A parser constructs trees over the input sentence by parsing with the source language projection of a synchronous $\mathrm{CFG}$, and each derivation induces translations in the target language (Chiang, 2007). However, in contrast to syntactic parsing, where linguistic intuitions can help elucidate the "right" tree structure for a grammatical sentence, no such intuitions are available for synchronous derivations, and so learning the "right" grammars is a central challenge.

Of course, learning synchronous grammars from parallel data is a widely studied problem (Wu, 1997; Blunsom et al., 2008; Levenberg et al., 2012, inter alia). However, there has been less exploration of learning rich non-terminal categories, largely because previous efforts to learn such categories have been coupled with efforts to learn derivation structures-a computationally formidable challenge. One popular approach has been to derive categories from source and/or target monolingual grammars (Galley et al., 2004; Zollmann and Venugopal, 2006; Hanneman and Lavie, 2013). While often successful, accurate parsers are not available in many languages: a more appealing approach is therefore to learn the category structure from the data itself.

In this work, we take a different approach to previous work in synchronous grammar induction by assuming that reasonable tree structures for a parallel corpus can be chosen heuristically, and then, fixing the trees (thereby enabling us to sidestep the worst of the computational issues), we learn non-terminal categories as latent variables to explain the distribution of these synchronous trees. This technique has a long history in monolingual parsing (Petrov et al., 2006; Liang et al., 2007; Cohen et al., 2014), where it reliably yields stateof-the-art phrase structure parsers based on generative models, but we are the first to apply it to translation.

We first generalize the concept of latent PCFGs to latent-variable SCFGs ( $(2)$. We then follow by a presentation of the tensor-based formulation for our parameters, a representation that makes it convenient to marginalize over latent states. Subsequently, two methods for parameter estimation are presented (§4): a spectral approach based on the method of moments, and an EM-based likelihood maximization. Results on a Chinese-English evaluation set ( $(5)$ indicate significant gains over baselines and point to the promise of using latentvariable synchronous grammars in conjunction with a smaller, simpler set of rules instead of unwieldy and bloated grammars extracted via existing heuristics, where a large number of contextindependent but un-generalizable rules are utilized. Hence, the hope is that this work pro- 
motes the move towards translation models that directly model the conditional likelihood of translation rules via (potentially feature-rich) latentvariable models which leverage information contained in the synchronous tree structure, instead of relying on a heuristic set of features based on empirical relative frequencies (Koehn et al., 2003) from non-hierarchical phrase-based translation.

\section{Latent-Variable SCFGs}

Before discussing parameter learning, we introduce latent-variable synchronous context-free grammars (L-SCFGs) and discuss an inference algorithm for marginalizing over latent states.

We extend the definition of L-PCFGs (Matsuzaki et al., 2005; Petrov et al., 2006) to synchronous grammars as used in machine translation (Chiang, 2007). A latent-variable SCFG (L$\mathrm{SCFG})$ is a 6-tuple $\left(\mathcal{N}, m, n_{s}, n_{t}, \pi, t\right)$ where:

- $\mathcal{N}$ is a set of non-terminal (NT) symbols in the grammar. For hierarchical phrase-based translation (HPBT), the set consists of only two symbols, $\mathbf{X}$ and a goal symbol $\mathbf{S}$.

- $[m]$ is the set of possible hidden states associated with NTs. Aligned pairs of NTs across the source and target languages share the same hidden state.

- $\left[n_{s}\right]$ is the set of source side words, i.e., the source-side vocabulary, with $\left[n_{s}\right] \cap \mathcal{N}=\emptyset$.

- $\left[n_{t}\right]$ is the set of target side words, i.e., the target-side vocabulary, with $\left[n_{t}\right] \cap \mathcal{N}=\emptyset$.

- The synchronous production rules compose a set $\mathcal{R}=\mathcal{R}_{0} \cup \mathcal{R}_{1} \cup \mathcal{R}_{2}$ :

- Arity 2 (binary) rules $\left(\mathcal{R}_{2}\right)$ : $a\left(h_{1}\right) \rightarrow\left\langle\alpha_{1} b\left(h_{2}\right) \alpha_{2} c\left(h_{3}\right) \alpha_{3}, \beta_{1} b\left(h_{2}\right) \beta_{2} c\left(h_{3}\right) \beta_{3}\right\rangle$

or

$a\left(h_{1}\right) \rightarrow\left\langle\alpha_{1} b\left(h_{2}\right) \alpha_{2} c\left(h_{3}\right) \alpha_{3}, \beta_{1} c\left(h_{2}\right) \beta_{2} b\left(h_{3}\right) \beta_{3}\right\rangle$

where $a, b, c \in \mathcal{N}, h_{1}, h_{2}, h_{3} \in[m]$, $\alpha_{1}, \alpha_{2}, \alpha_{3} \in\left[n_{s}\right]^{*}$ and $\beta_{1}, \beta_{2}, \beta_{3} \in\left[n_{t}\right]^{*}$.

- Arity 1 (unary) rules $\left(\mathcal{R}_{1}\right)$ :

$$
a\left(h_{1}\right) \rightarrow\left\langle\alpha_{1} b\left(h_{2}\right) \alpha_{2}, \beta_{1} b\left(h_{2}\right) \beta_{2}\right\rangle
$$

where $a, b \in \mathcal{N}, h_{1}, h_{2} \in[m], \alpha_{1}, \alpha_{2} \in\left[n_{s}\right]^{*}$ and $\beta, \beta_{2} \in\left[n_{t}\right]^{*}$.

- Pre-terminal rules $\left(\mathcal{R}_{0}\right): a\left(h_{1}\right) \rightarrow\langle\alpha, \beta\rangle$ where $a \in \mathcal{N}, \alpha \in\left[n_{t}\right]^{*}$ and $\beta \in\left[n_{s}\right]^{*}$.
Each of these rules is associated with a probability $t\left(a\left(h_{1}\right) \rightarrow \gamma \mid a, h_{1}\right)$ where $\gamma$ is the righthand side (RHS) of the rule.

- For $a \in \mathcal{N}, h \in[m], \pi(a, h)$ is a parameter specifying the root probability of $a(h)$.

A skeletal tree (s-tree) for a sentence is the set of rules in the synchronous derivation of that sentence, without any additional latent state information or decoration. A full tree consists of an stree $r_{1}, \ldots, r_{N}$ together with values $h_{1}, \ldots, h_{N}$ for every NT in the tree. An important point to keep in mind in comparison to L-PCFGs is that the right-hand side (RHS) non-terminals of synchronous rules are aligned pairs across the source and target languages.

In this work, we refine the one-category grammar introduced by Chiang (2007) for HPBT in order to learn additional latent NT categories. Thus, the following discussion is restricted to these kinds of grammars, although the method is equally applicable in other scenarios, e.g., the extended treeto-string transducer (xRs) formalism (Huang et al., 2006; Graehl et al., 2008) commonly used in syntax-directed translation, and phrase-based MT (Koehn et al., 2003).

Marginal Inference with L-SCFGs. For a parameter $t$ of rule $r$, the latent state $h_{1}$ attached to the left-hand side (LHS) NT of $r$ is associated with the outside tree for the sub-tree rooted at the LHS, and the states attached to the RHS NTs are associated with the inside trees of that NT. Since we do not assume conditional independence of these states, we need to consider all possible interactions, which can be compactly represented as a $3^{\text {rd }}$-order tensor in the case of a binary rule, a matrix (i.e., a $2^{\text {nd }}$-order tensor) for unary rules, and a vector for pre-terminal (lexical) rules. Preferences for certain outside-inside tree combinations are reflected in the values contained in these tensor structures. In this manner, we intend to capture interactions between non-local context of a phrase, which can typically be represented via features defined over outside trees of the node spanning the phrase, and the interior context, correspondingly defined via features over the inside trees. We refer to these tensor structures collectively as $C^{r}$ for rules $r \in \mathcal{R}$, which encompass the parameters $t$.

For $r \in \mathcal{R}_{0}: C^{r} \in \mathbb{R}^{m \times 1}$; similarly for $r \in \mathcal{R}_{1}: C^{r} \in \mathbb{R}^{m \times m}$ and $r \in \mathcal{R}_{2}: C^{r} \in$ $\mathbb{R}^{m \times m \times m}$. We also maintain a vector $C^{\mathbf{S}} \in \mathbb{R}^{1 \times m}$ corresponding to the parameters $\pi(\mathbf{S}, h)$ for the 


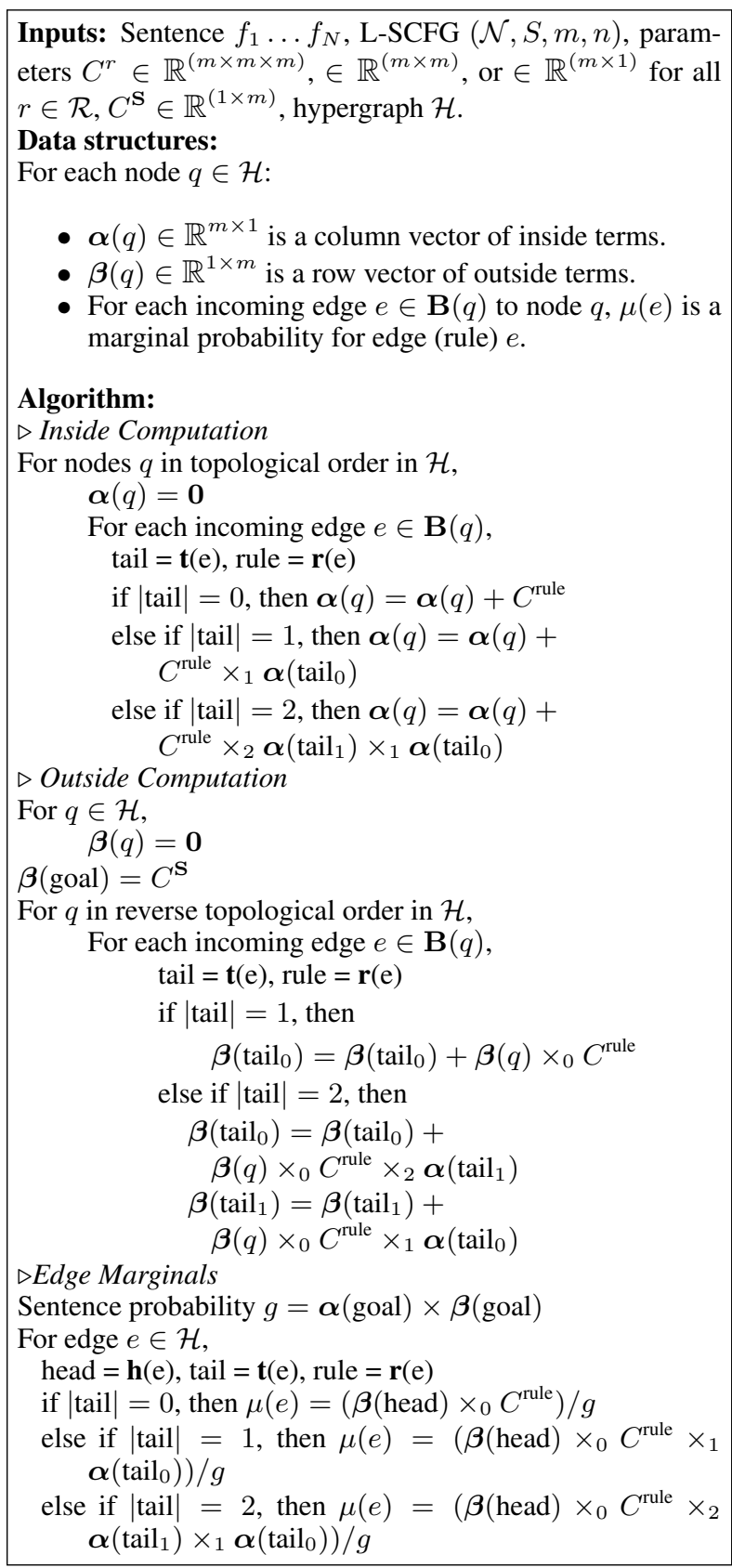

Figure 1: The tensor form of the hypergraph insideoutside algorithm, for calculation of rule marginals $\mu(e)$. A slight simplification in the marginal computation yields NT marginals for spans $\mu(\mathbf{X}, i, j)$. B(q) returns the incoming hyperedges for node $q$, and $\mathbf{h}(\mathrm{e}), \mathbf{t}(\mathrm{e}), \mathbf{r}(\mathrm{e})$ return the head node, tail nodes, and rule for hyperedge $e$.

goal node (root). These parameters participate in tensor-vector operations: a $3^{\text {rd }}$-order tensor $C^{r_{2}}$ can be multiplied along each of its three modes $\left(\times_{0}, \times_{1}, \times_{2}\right)$, and if multiplied by an $m \times 1$ vector, will produce an $m \times m$ matrix. ${ }^{1}$ Note that matrix multiplication can be represented by $\times_{1}$ when multiplying on the right and $\times_{0}$ when multiplying on the left of the matrix. The decoder computes marginal probabilities for each skeletal rule in the

\footnotetext{
${ }^{1}$ This operation is sometimes called a contraction.
}

parse forest of a source sentence by marginalizing over the latent states, which in practice corresponds to simple tensor-vector products. This operation is not dependent on the manner in which the parameters were estimated.

Figure 1 presents the tensor version of the inside-outside algorithm for decoding L-SCFGs. The algorithm takes as input the parse forest of the source sentence represented as a hypergraph (Klein and Manning, 2001), which is computed using a bottom-up parser with Earley-style rules similar to the algorithm in Chiang (2007). Hypergraphs are a compact way to represent a forest of multiple parse trees. Each node in the hypergraph corresponds to an NT span, and can have multiple incoming and outgoing hyperedges. Hyperedges, which connect one or more tail nodes to a single head node, correspond exactly to rules, and tail or head nodes correspond to children (RHS NTs) or parent (LHS NT). The function $\mathbf{B}(\mathrm{q})$ returns all incoming hyperedges to a node q, i.e., all rules such that the LHS NT of the rule corresponds to the NT span of the node q. The algorithm computes inside and outside probabilities over the hypergraph using the tensor representations, and converts these probabilities to marginal rule probabilities. It is similar to the version presented in Cohen et al. (2014), but adapted to hypergraph parse forests.

The complexity of this decoding algorithm is $\mathcal{O}\left(n^{3} m^{3}|G|\right)$ where $n$ is the length of the input sentence, $m$ is the number of latent states, and $|G|$ is the number of production rules in the grammar without latent-variable annotations (i.e., $m=1$ ). ${ }^{2}$ The bulk of the computation is a series of tensorvector products of relatively small size (each dimension is of length $m$ ), which can be computed very quickly and in parallel. The tensor computations can be significantly sped up using techniques described by Cohen and Collins (2012), so that they are linear in $m$ and not cubic.

\section{Derivation Trees for Parallel Sentences}

To estimate the parameters $t$ and $\pi$ of an LSCFG (discussed in detail in the next section), we assume the existence of a dataset composed of synchronous s-trees, which can be acquired from word alignments. Normally in phrase-based translation models, we consider all possible phrase

\footnotetext{
${ }^{2}$ In practice, the term $m^{3}|G|$ can be replaced with a smaller term, which separates the rules in $G$ by the number of NTs on the RHS. This idea relates to the notion of "effective grammar size" which we discuss in $\S 5$.
} 
pairs consistent with the word alignments and estimate features based on surface statistics associated with the phrase pairs or rules. The weights of these features are then learned using a discriminative training algorithm (Och, 2003; Chiang, 2012, inter alia). In contrast, in this work we restrict the number of possible synchronous derivations for each sentence pair to just one; thus, derivation forests do not have to be considered, making parameter estimation more tractable. ${ }^{3}$

To achieve this objective, for each sentence in the training data we extract the minimal set of synchronous rules consistent with the word alignments, as opposed to the composed set of rules (Galley et al., 2006). Composed rules are ones that can be formed from smaller rules in the grammar; with these rules, there are multiple synchronous trees consistent with the alignments for a given sentence pair, and thus the total number of applicable rules can be combinatorially larger than if we just consider the set of rules that cannot be formed from other rules, namely the minimal rules. The rule types across all sentence pairs are combined to form a minimal grammar. ${ }^{4}$ To extract a set of minimal rules, we use the linear-time extraction algorithm of Zhang et al. (2008). We give a rough description of their method below, and refer the reader to the original paper for additional details.

The algorithm returns a complete minimal derivation tree for each word-aligned sentence pair, and generalizes an approach for finding all common intervals (pairs of phrases such that no word pair in the alignment links a word inside the phrase to a word outside the phrase) between two permutations (Uno and Yagiura, 2000) to sequences with many-to-many alignment links between the two sides, as in word alignment. The key idea is to encode all phrase pairs of a sentence alignment in a tree of size proportional to the source sentence length, which they call the normalized decomposition tree. Each node corresponds to a phrase pair, with larger phrase spans represented by higher nodes in the tree. Constructing the tree is analogous to finding common intervals in two permutations, a property that they leverage to propose a linear-time algorithm for tree

\footnotetext{
${ }^{3}$ For future work, we will consider efficient algorithms for parameter estimation over derivation forests, since there may be multiple valid ways to explain the sentence pair via a synchronous tree structure.

${ }^{4}$ Table 2 presents a comparison of grammar sizes for our experiments $(\S 5.1)$.
}

extraction. Converting the tree to a set of minimal SCFG rules for the sentence pair is straightforward, by replacing nodes corresponding to spans with lexical items or NTs in a bottom-up manner. ${ }^{5}$

By using minimal rules as a starting point instead of the traditional heuristically-extracted rules (Chiang, 2007) or arbitrary compositions of minimal rules (Galley et al., 2006), we are also able to explore the transition from minimal rules to composed ones in a principled manner by encoding contextual information through the latent states. Thus, a beneficial side effect of our refinement process is the creation of more contextspecific rules without increasing the overall size of the baseline grammar, instead holding this information in our parameters $C^{r}$.

\section{Parameter Estimation for L-SCFGs}

We explore two methods for estimating the parameters $C^{r}$ of the model: a likelihood-maximization approach based on EM (Dempster et al., 1977), and a spectral approach based on the method of moments (Hsu et al., 2009; Cohen et al., 2014), where we identify a subspace using a singular value decomposition (SVD) of the cross-product feature space between inside and outside trees and estimate parameters in this subspace.

Figure 2 presents a side-by-side comparison of the two algorithms, which we discuss in this section. In the spectral approach, we base our parameter estimates on low-rank representations of moments of features, while EM explicitly maximizes a likelihood criterion. The parameter estimation algorithms are relatively similar, but in lieu of sparse feature functions in the spectral case, EM uses partial counts estimated with the current set of parameters. The nature of EM allows it to be susceptible to local optima, while the spectral approach comes with guarantees on obtaining the global optimum (Cohen et al., 2014). Lastly, computing the SVD and estimating parameters in the low-rank space is a one-shot operation, as opposed to the iterative procedure of EM, and therefore is much more computationally efficient.

\subsection{Estimation with Spectral Method}

We generalize the parameter estimation algorithm presented in Cohen et al. (2013) to the syn-

\footnotetext{
${ }^{5}$ We filtered rules with arity 3 and above (i.e., containing more than 3 NTs on the RHS). While the L-SCFG formalism is perfectly capable of handling such cases, it would have resulted in higher order tensors for our parameter structures.
} 


\begin{abstract}
Inputs:
Training examples $\left(r^{(i)}, t^{(i, 1)}, t^{(i, 2)}, t^{(i, 3)}, o^{(i)}, b^{(i)}\right)$ for $i \in\{1 \ldots M\}$, where $r^{(i)}$ is a context free rule; $t^{(i, 1)}, t^{(i, 2)}$, and $t^{(i, 3)}$ are inside trees; $o^{(i)}$ is an outside tree; and $b^{(i)}=1$ if the rule is at the root of tree, 0 otherwise. A function $\phi$ that maps inside trees $t$ to feature-vectors $\phi(t) \in \mathbb{R}^{d}$. A function $\psi$ that maps outside trees $o$ to feature-vectors $\psi(o) \in \mathbb{R}^{d^{\prime}}$.

Algorithm:

$\triangleright$ Step 0: Singular Value Decomposition
\end{abstract}

- Compute the SVD of Eq. 1 to calculate matrices $\hat{U} \in \mathbb{R}^{(d \times m)}$ and $\hat{V} \in \mathbb{R}^{\left(d^{\prime} \times m\right)}$.

$\triangleright$ Step 1: Projection

$$
\begin{aligned}
& Y(t)=U^{\top} \phi(t) \\
& Z(o)=\Sigma^{-1} V^{\top} \psi(o)
\end{aligned}
$$

$\triangleright$ Step 2: Calculate Correlations

$$
\hat{E}^{r}= \begin{cases}\frac{\sum_{o \in Q^{r}} Z(o)}{\left|Q^{r}\right|} & \text { if } r \in \mathcal{R}_{0} \\ \frac{\sum_{(o, t) \in Q^{r} Z(o) \otimes Y(t)}\left|Q^{r}\right|}{\sum_{\left(o, t^{2}, t^{3}\right) \in Q^{r}} Z(o) \otimes Y\left(t^{2}\right) \otimes Y\left(t^{3}\right)} & \text { if } r \in \mathcal{R}_{1} \\ \frac{Q^{r} \mid}{2} & \text { if } r \in \mathcal{R}_{2}\end{cases}
$$

$Q^{r}$ is the set of outside-inside tree triples for binary rules, outside-inside tree pairs for unary rules, and outside trees for pre-terminals.

$\triangleright$ Step 3: Compute Final Parameters

- For all $r \in \mathcal{R}$,

$$
\hat{C}^{r}=\frac{\operatorname{count}(r)}{M} \times \hat{E}^{r}
$$

- For all $r^{(i)} \in\{1, \ldots, M\}$ such that $b^{(i)}$ is 1 ,

$$
\hat{C}^{\mathbf{S}}=\hat{C}^{\mathbf{S}}+\frac{Y\left(t^{(i, 1)}\right)}{\left|Q^{\mathbf{S}}\right|}
$$

$Q^{\mathbf{S}}$ is the set of trees at the root.

\section{Inputs:}

Training examples $\left(r^{(i)}, t^{(i, 1)}, t^{(i, 2)}, t^{(i, 3)}, o^{(i)}, b^{(i)}\right)$ for $i \in$ $\{1 \ldots M\}$, where $r^{(i)}$ is a context free rule; $t^{(i, 1)}, t^{(i, 2)}$, and $t^{(i, 3)}$ are inside trees; $o^{(i)}$ is an outside tree; $b^{(i)}=1$ if the rule is at the root of tree, 0 otherwise; and MAX_ITERATIONS.

Algorithm:

$\triangle$ Step 0: Parameter Initialization

For rule $r \in \mathcal{R}$,

- if $r \in \mathcal{R}_{0}$ : initialize $\hat{C}^{r} \in \mathbb{R}^{m \times 1}$

- if $r \in \mathcal{R}_{1}$ : initialize $\hat{C}^{r} \mathbb{R}^{m \times m}$

- if $r \in \mathcal{R}_{2}$ : initialize $\hat{C}^{r} \mathbb{R}^{m \times m \times m}$

Initialize $\hat{C}^{\mathbf{s}} \in \mathbb{R}^{m \times 1}$

$\hat{C}_{0}^{r}=\hat{C}^{r}, \hat{C}_{0}^{\mathbf{S}}=\hat{C}^{\mathbf{S}}$

For iteration $t=1, \ldots$, MAX_ITERATIONS,

- Expectation Step:

$\triangleright$ Estimate $Y$ and $Z$

Compute partial counts and total tree probabilities $g$ for all $t$ and $o$ using Fig. 1 and parameters $\hat{C}_{t-1}^{r}, \hat{C}_{t-1}^{\mathbf{S}}$.

$\triangleright$ Calculate Correlations

$\hat{E}^{r}= \begin{cases}\sum_{o, g \in Q^{r}} \frac{Z(o)}{g} & \text { if } r \in \mathcal{R}_{0} \\ \sum_{(o, t, g) \in Q^{r}} \frac{Z(o) \otimes Y(t)}{g} & \text { if } r \in \mathcal{R}_{1} \\ \sum_{\left(o, t^{2}, t^{3}, g\right) \in Q^{r}} \frac{Z(o) \otimes Y\left(t^{2}\right) \otimes Y\left(t^{3}\right)}{g} & \text { if } r \in \mathcal{R}_{2}\end{cases}$

$\triangleright$ Update Parameters

For all $r \in \mathcal{R}, \hat{C}_{t}^{r}=\hat{C}_{t-1}^{r} \odot \hat{E}^{r}$

For all $r^{(i)} \in\{1, \ldots, M\}$ such that $b^{(i)}$ is 1 , $\hat{C}_{t}^{\mathbf{S}}=\hat{C}_{t}^{\mathbf{S}}+\left(\hat{C}_{t-1}^{\mathbf{S}} \odot Y\left(r^{(i)}\right)\right) / g$

$Q^{\mathrm{S}}$ is the set of trees at the root.

- Maximization Step

$$
\begin{aligned}
& \text { if } r \in \mathcal{R}_{0}: \forall h_{1}: \hat{C}^{r}\left(h_{1}\right)=\frac{\hat{C}^{r}\left(h_{1}\right)}{\sum_{r^{\prime}=r} \sum_{h_{1}} \hat{C}^{r^{\prime}}\left(h_{1}\right)} \\
& \text { if } r \in \mathcal{R}_{1}: \forall h_{1}, h_{2}: \hat{C}^{r}\left(h_{1}, h_{2}\right)= \\
& \frac{\hat{C}^{r}\left(h_{1}, h_{2}\right)}{\sum_{r^{\prime}=r} \sum_{h_{2}} \hat{C}^{r^{\prime}}\left(h_{1}, h_{2}\right)} \\
& \text { if } r \in \mathcal{R}_{2}: \forall h_{1}, h_{2}, h_{3}: \hat{C}^{r}\left(h_{1}, h_{2}, h_{3}\right)= \\
& \frac{\hat{C}^{r}\left(h_{1}, h_{2}, h_{3}\right)}{\sum_{r^{\prime}=r} \sum_{h_{2}, h_{3}} \hat{C}^{r^{\prime}}\left(h_{1}, h_{2}, h_{3}\right)} \\
& \text { if } \operatorname{LHS}(r)=\mathbf{S}: \quad \forall h_{1}: \hat{C}^{r}\left(h_{1}\right)= \\
& \frac{\hat{C}^{r}\left(h_{1}\right)}{\sum_{r^{\prime}=r} \sum_{h_{1}} \hat{C}^{r^{\prime}}\left(h_{1}\right)}
\end{aligned}
$$

(b) The EM-based algorithm for estimating parameters of an LSCFG.

(a) The spectral learning algorithm for estimating parameters of an L-SCFG.

Figure 2: The two parameter estimation algorithms proposed for L-SCFGs; (a) method of moments; (b) expectation maximization. $\odot$ is the element-wise multiplication operator.

chronous or bilingual case. The central concept of the spectral parameter estimation algorithm is to learn an $m$-dimensional representation of inside and outside trees by defining these trees in terms of features, in combination with a projection step (SVD), with the hope being that the lowerdimensional space captures the syntactic and se- mantic regularities among rules from the sparse feature space. Every NT in an s-tree has an associated inside and outside tree; the inside tree contains the entire sub-tree at and below the NT, and the outside tree is everything else in the synchronous s-tree except the inside tree. The inside feature function $\phi$ maps the domain of inside tree 
fragments to a $d$-dimensional Euclidean space, and the outside feature function $\psi$ maps the domain of outside tree fragments to a $d^{\prime}$-dimensional space. The specific features we used are discussed in $\S 5.2$.

Let $\mathcal{O}$ be the set of all tuples of inside-outside trees in our training corpus, whose size is equivalent to the number of rule tokens (occurrences in the corpus) $M$, and let $\phi(t) \in \mathbb{R}^{d \times 1}, \psi(o) \in \mathbb{R}^{d^{\prime} \times 1}$ be the inside and outside feature functions for inside tree $t$ and outside tree $o$. By computing the outer product $\otimes$ between the inside and outside feature vectors for each pair and aggregating, we obtain the empirical inside-outside feature covariance matrix:

$$
\hat{\Omega}=\frac{1}{|\mathcal{O}|} \sum_{(o, t) \in \mathcal{O}} \phi(t)(\psi(o))^{\top}
$$

If $m$ is the desired latent space dimension, we compute an $m$-rank truncated SVD of the empirical covariance matrix $\hat{\Omega} \approx U \Sigma V^{\top}$, where $U \in$ $\mathbb{R}^{d \times m}$ and $V \in \mathbb{R}^{d^{\prime} \times m}$ are the matrices containing the left and right singular vectors, and $\Sigma \in \mathbb{R}^{m \times m}$ is a diagonal matrix containing the $m$-largest singular values along its diagonal.

Figure 2a provides the remaining steps in the algorithm. The $M$ training examples are obtained by considering all nodes in all of the synchronous s-trees given as input. In step 1, for each inside and outside tree, we project its high-dimensional representation to the $m$-dimensional latent space. Using the $m$-dimensional representations for inside and outside trees, in step 2 for each rule type $r$ we compute the covariance between the inside tree vectors and the outside tree vector using the tensor product, a generalized outer product to compute covariances between more than two random vectors. For binary rules, with two child inside vectors and one outside vector, the result $\hat{E}^{r}$ is a 3-mode tensor; for unary rules, a regular matrix, and for pre-terminal rules with no right-hand side non-terminals, a vector. The final parameter estimate is then the associated tensor/matrix/vector, scaled by the maximum likelihood estimate of the rule $r$, as in step 3 .

The corresponding theoretical guarantees from Cohen et al. (2014) can also be generalized to the synchronous case. $\hat{\Omega}$ is an empirical estimate of the true covariance matrix $\Omega$, and if $\Omega$ has rank $m$, then the marginals computed using the spectrally-estimated parameters will converge to the true marginals, with the sample complexity for convergence inversely proportional to a polynomial function of the $m^{\text {th }}$ largest singular value of $\Omega$.

\subsection{Estimation with EM}

A likelihood maximization approach can also be used to learn the parameters of an L-SCFG. Parameters are initialized by sampling each parameter value $\hat{C}^{r}\left(h_{1}, h_{2}, h_{3}\right)$ from the interval $[0,1]$ uniformly at random. ${ }^{6}$ We first decode the training corpus using an existing set of parameters to compute the inside and outside probability vectors associated with NTs for every rule in each s-tree, constrained to the tree structure of the training example. These probabilities can be computed using the decoding algorithm in Figure 1 (where $\boldsymbol{\alpha}$ and $\boldsymbol{\beta}$ correspond to the inside and outside probabilities respectively), except the parse forest consists of a single tree only. These vectors represent partial counts over latent states. We then define functions $Y$ and $Z$ (analogous to the spectral case) which map inside and outside tree instances to $m$-dimensional vectors containing these partial counts. In the spectral case, $Y$ and $Z$ are estimated just once, while in the case of EM they have to be re-estimated at each iteration.

The expectation step thus consists of computing the partial counts of inside and outside trees $t$ and $o$, i.e., recovering the functions $Y$ and $Z$, and updating parameters $C^{r}$ by computing correlations, which involves summing over partial counts (across all occurrences of a rule in the corpus). Each partial count's contribution is divided by a normalization factor $g$, which is the total probability of the tree which $t$ or $o$ is part of. Note that unlike the spectral case, there is a specific normalization factor for each inside-outside tuple. Lastly, the correlations are scaled by the existing parameter estimates.

To obtain the next set of parameters, in the maximization step we normalize $\hat{C}^{r}$ for $r \in \mathcal{R}$ such that for every $h_{1}, \sum_{r^{\prime}=r, h_{2}, h_{3}} \hat{C}^{r^{\prime}}\left(h_{1}, h_{2}, h_{3}\right)=1$ for $r \in \mathcal{R}_{2}, \sum_{r^{\prime}=r, h_{2}} \hat{C}^{r^{\prime}}\left(h_{1}, h_{2}\right)=1$ for $r \in \mathcal{R}_{1}$, and $\sum_{r^{\prime}=r, h_{2}} \hat{C}^{r^{\prime}}\left(h_{2}\right)=1$ for $r \in \mathcal{R}_{0}$. We also normalize the root rule parameters $\hat{C}^{r}$ where $\operatorname{LHS}(r)=\mathbf{S}$. It is also possible to add sparse, overlapping features to an EM-based estimation

\footnotetext{
${ }^{6}$ In our experiments, we also tried the initialization scheme described in Matsuzaki et al. (2005), but found that it provided little benefit.
} 
procedure (Berg-Kirkpatrick et al., 2010) and we leave this extension for future work.

\section{Experiments}

The goal of the experimental section is to evaluate the performance of the latent-variable SCFG in comparison to a baseline without any additional NT annotations (MIN-GRAMMAR), and to compare the performance of the two parameter estimation algorithms. We also compare L-SCFGs to a HiERo baseline (Chiang, 2007). The language pair of evaluation is Chinese-English (ZH-EN).

We score translations using BLEU (Papineni et al., 2002). The latent-variable model is integrated into the standard MT pipeline by computing marginal probabilities for each rule in the parse forest of a source sentence using the algorithm in Figure 1 with the parameters estimated through the algorithms in Figure 2, and is added as a feature for the rule during MERT (Och, 2003). These probabilities are conditioned on the LHS $(\mathbf{X})$, and are thus joint probabilities for a source-target RHS pair. We also write out as features the conditional relative frequencies $\hat{P}(e \mid f)$ and $\hat{P}(f \mid e)$ as estimated by our latent-variable model, i.e., conditioned on the source and target RHS.

Overall, we find that both the spectral and the EM-based estimators improve upon a minimal grammar baseline with only a single category, but the spectral approach does better. In fact, it matches the performance of the standard HIERO baseline, despite learning on top of a minimal grammar.

\subsection{Data and Baselines}

The ZH-EN data is the BTEC parallel corpus (Paul, 2009); we combine the first and second development sets in one, and evaluate on the third development set. The development and test sets are evaluated with 16 references. Statistics for the data are shown in Table 1. We used the CDEC decoder (Dyer et al., 2010) to extract word alignments and the baseline hierarchical grammars, MERT tuning, and decoding. We used a 4-gram language model built from the target-side of the parallel training data. The Python-based implementation of the tensor-based decoder, as well as the parameter estimation algorithms is available at github.com/asaluja/spectral-scfg/.

The baseline HIERO system uses a grammar extracted by applying the commonly used heuris-

\begin{tabular}{lr}
\hline & ZH-EN \\
\hline TRAIN (SRC) & $334 \mathrm{~K}$ \\
TRAIN (TGT) & $366 \mathrm{~K}$ \\
DEV (SRC) & $7 \mathrm{~K}$ \\
DEV (TGT) & $7.6 \mathrm{~K}$ \\
TEST (SRC) & $3.8 \mathrm{~K}$ \\
TEST (TGT) & $3.9 \mathrm{~K}$
\end{tabular}

Table 1: Corpus statistics (in words). For the target DEV and TEST statistics, we take the first reference.

tics (Chiang, 2007). Each rule is decorated with two lexical and phrasal features corresponding to the forward $(e \mid f)$ and backward $(f \mid e)$ conditional $\log$ frequencies, along with the log joint frequency $(e, f)$, the log frequency of the source phrase $(f)$, and whether the phrase pair or the source phrase is a singleton. Weights for the language model (and language model OOV), glue rule, and word penalty are also tuned. The MIN-GRAMMAR baseline $^{7}$ maintains the same set of weights.

\begin{tabular}{lr}
\hline Grammar & Number of Rules \\
\hline HIERO & $1.69 \mathrm{M}$ \\
MIN-GRAMMAR & $59 \mathrm{~K}$ \\
LV $m=1$ & $27.56 \mathrm{~K}$ \\
LV $m=8$ & $3.18 \mathrm{M}$ \\
LV $m=16$ & $22.22 \mathrm{M}$
\end{tabular}

Table 2: Grammar sizes for the different systems; for the latent-variable models, effective grammar sizes are provided.

Grammar sizes are presented in Table 2. For the latent-variable models, we provide the effective grammar size, where the number of NTs on the RHS of a rule is taken into account when computing the grammar size, by assuming each possible latent variable configuration amongst the NTs generates a different rule. Furthermore, all singletons are mapped to the OOV rule, while we include singletons in MIN-GRAMMAR. ${ }^{8}$ Hence, effective grammar size can be computed as $m(1+$ $\left.\left|\mathcal{R}_{0}^{>1}\right|\right)+m^{2}\left|\mathcal{R}_{1}\right|+m^{3}\left|\mathcal{R}_{2}\right|$, where $\mathcal{R}_{0}^{>1}$ is the set of pre-terminal rules that occur more than once.

\subsection{Spectral Features}

We use the following set of sparse, binary features in the spectral learning process:

\footnotetext{
${ }^{7}$ Code to extract the minimal derivation trees is available at www.cs.rochester.edu/u/gildea/mt/.

${ }^{8}$ This OOV mapping is done so that the latent-variable model can handle unknown tokens.
} 
- Rule Indicator. For the inside features, we consider the rule production containing the current non-terminal on the left-hand side, as well as the rules of the children (distinguishing between left and right children for binary rules). For the outside features, we consider the parent rule production along with the rule production of the sibling (if it exists).

- Lexical. for both the inside and outside features, any lexical items that appear in the rule productions are recorded. Furthermore, we consider the first and last words of spans (left and right child spans for inside features, distinguishing between the two if both exist, and sibling span for outside features). Source and target words are treated separately.

- Length. the span length of the tree and each of its children for inside features, and the span length of the parent and sibling for outside features.

In our experiments, we instantiated a total of 170,000 rule indicator features, 155,000 lexical features, and 80 length features.

\subsection{Chinese-English Experiments}

Table 3 presents a comprehensive evaluation of the ZH-EN experimental setup. The first section consists of the various baselines we consider. In addition to the aforementioned baselines, we evaluated a setup where the spectral parameters simply consist of the joint maximum likelihood estimates of the rules. This baseline should perform en par with MIN-GRAMMAR, which we see is the case on the development set. The performance on the test set is better though, primarily because we also include the reverse log relative frequency $(f \mid e)$ computed from the latent-variable model as an additional feature in MERT. Furthermore, in line with previous work (Galley et al., 2006) which compares minimal and composed rules, we find that minimal grammars take a hit of more than 2.5 BLEU points on the development set, compared to composed (HIERO) grammars. The $m=1$ spectral baseline with only rule indicator features performs slightly better than the minimal grammar baseline, since it overtly takes into account insideoutside tree combination preferences in the parameters, but improvement is minimal with one latent state naturally and the performance on the test set is in line with the MLE baseline.

On top of the baselines, we looked at a number

\begin{tabular}{|l|lrr|}
\hline \multirow{5}{*}{ Baselines } & Setup & Dev & Test \\
\hline & HIERO & 46.08 & 55.31 \\
& MIN- & 43.38 & 51.78 \\
& GRAMMAR & & \\
& MLE & 43.24 & 52.80 \\
\hline \multirow{5}{*}{ Spectral } & $m=1$ RI & 44.18 & 52.62 \\
& $m=8$ RI & 44.60 & 53.63 \\
& $m=16$ RI & 46.06 & 55.83 \\
& $m=16$ RI+Lex+Sm & 46.08 & 55.22 \\
& $m=16$ RI+Lex+Len & 45.70 & 55.29 \\
& $m=24$ RI+Lex & 43.00 & 51.28 \\
& $m=32$ RI+Lex & 43.06 & 52.16 \\
\hline \multirow{2}{*}{ EM } & $m=8$ & $40.53(0.2)$ & $49.78(0.5)$ \\
& $m=16$ & $42.85(0.2)$ & $52.93(0.9)$ \\
& $m=32$ & $41.07(0.4)$ & $49.95(0.7)$ \\
\hline
\end{tabular}

Table 3: Results for the $\mathrm{ZH}-\mathrm{EN}$ corpus, comparing across the baselines and the two parameter estimation techniques. RI, Lex, and Len correspond to the rule indicator, lexical, and length features respectively, and Sm denotes smoothing. For the EM experiments, we selected the best scoring iteration by tuning weights for parameters obtained after 25 iterations and evaluating other parameters with these weights. Results for EM are averaged over 5 starting points, with standard deviation given in parentheses. Spectral, EM, and MLE performances compared to the MIN-GRAMMAR baseline are statistically significant $(p<0.01)$.

of feature combinations and latent states for the spectral and EM-estimated latent-variable models. For the spectral models, we tuned MERT parameters separately for each rank on a set of parameters estimated from rule indicator features only; subsequent variations within a given rank, e.g., the addition of lexical or length features or smoothing, were evaluated with the same set of rank-specific weights from MERT. For EM, we ran parameter estimation with 5 randomly initialized starting points for 50 iterations; we tuned the MERT parameters with EM parameters obtained after $25^{\text {th }}$ iterations. Similar to the spectral experiments, we fixed the MERT weight values and evaluated BLEU performance with parameters after every 5 iterations and chose the iteration with the highest score on the development set. The results are averaged over the 5 initializations, with standard deviation in parentheses.

Firstly, we can see a clear dependence on rank, with peak performance for the spectral and EM models occurring at $m=16$. In this instance, the spectral model roughly matches the performance of the HIERO baseline, but it only uses rules extracted from a minimal grammar, whose size is a fraction of the HIERO grammar. The gains seem to level off at this rank; additional ranks seem to add noise to the parameters. Feature-wise, additional lexical and length features add little, prob- 
ably because much of this information is encapsulated in the rule indicator features. For EM, $m=16$ outperforms the minimal grammar baseline, but is not at the level of the spectral results. All EM, spectral, and MLE results are statistically significant $(p<0.01)$ with respect to the MINGRAMMAR baseline (Zhang et al., 2004), and the improvement over the HIERO baseline achieved by the $m=16$ rule indicator configuration is also statistically significant.

The two estimation algorithms differ significantly in their estimation time. Given a feature covariance matrix, the spectral algorithm (SVD, which was done with Matlab, and correlation computation steps) for $m=16$ took 7 minutes, while the EM algorithm took 5 minutes for each iteration with this rank.

\subsection{Analysis}

Figure 3 presents a comparison of the nonterminal span marginals for two sentences in the development set. We visualize these differences through a heat map of the CKY parse chart, where the starting word of the span is on the rows, and the span end index is on the columns. Each cell is shaded to represent the marginal of that particular non-terminal span, with higher likelihoods in blue and lower likelihoods in red.

For the most part, marginals at the leaves (i.e., pre-terminal marginals) tend to score relatively similarly across different setups. Higher up in the chart, the latent SCFG marginals look quite different than the MLE parameters. Most noticeably, spans starting at the beginning of the sentence are much more favored. It is these rules that allow the right translation to be preferred since the MLE chooses not to place the object of the sentence in the subject's span. However, the spectral parameters seem to discriminate between these higherlevel rules better than EM, which scores spans starting with the first word uniformly highly. Another interesting point is that the range of likelihoods is much larger in the EM case compared to the MLE and spectral variants. For the second sentence (row), the 1-best hypothesis produced by all systems are the same, but the heat map accentuates the previous observation.

\section{Related Work}

The goal of refining single-category HPBT grammars or automatically learning the NT categories in a grammar, instead of relying on noisy parser outputs, has been explored from several different angles in the MT literature. Blunsom et al. (2008) present a Bayesian model for synchronous grammar induction, and place an appropriate nonparametric prior on the parameters. However, their starting point is to estimate a synchronous grammar with multiple categories from parallel data (using the word alignments as a prior), while we aim to refine a fixed grammar with additional latent states. Furthermore, their estimation procedure is extremely expensive and is restricted to learning up to five NT categories, via a series of mean-field approximations.

Another approach is to explicitly attach a realvalued vector to each NT: Huang et al. (2010) use an external source-language parser for this purpose and score rules based on the similarity between a source sentence parse and the information contained in this vector, which explicitly requires the integration of a good-quality source-language parser. The EM-based algorithm that we propose here is similar to what they propose, except that we need to handle tensor structures. Mylonakis and Sima' an (2011) select among linguistically motivated non-terminal labels with a cross-validated version of EM. Although they consider a restricted hypothesis space, they do marginalize over different derivations therefore their inside-outside algorithm is $\mathcal{O}\left(n^{6}\right)$. In the syntax-directed translation literature, there have been efforts to relax or coarsen the hard labels provided by a syntactic parser in an automatic manner to promote parameter sharing (Venugopal et al., 2009; Hanneman and Lavie, 2013), which is the complement of our aim in this paper.

The idea of automatically learned grammar refinements comes from the monolingual parsing literature, where phenomena like head lexicalization can be modeled through latent variables. Matsuzaki et al. (2005) look at a likelihood-based method to split the NT categories of a grammar into a fixed number of sub-categories, while Petrov et al. (2006) learn a variable number of sub-categories per NT. The latter's extension may be useful for finding the optimal number of latent states from the data in our case.

The question of whether we can incorporate additional contextual information in minimal rule grammars in MT via auxiliary models instead of using longer, composed rules has been investigated before as well. $n$-gram translation mod- 


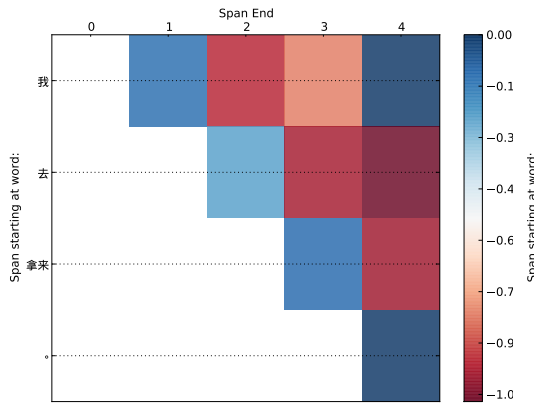

I go away .

(a) MLE

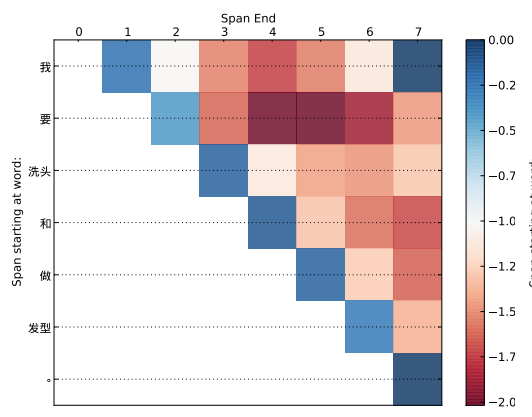

I'd like a shampoo and style .

(d) MLE

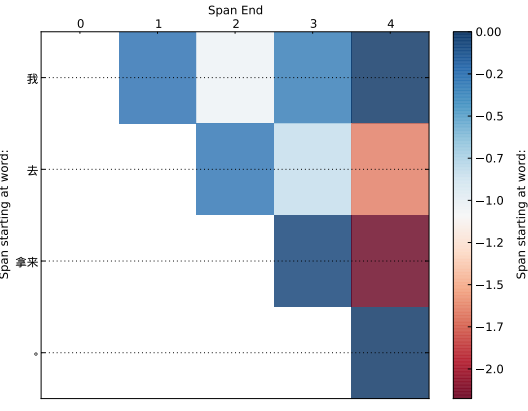

I 'll bring it .

(b) Spectral $m=16 \mathrm{RI}$

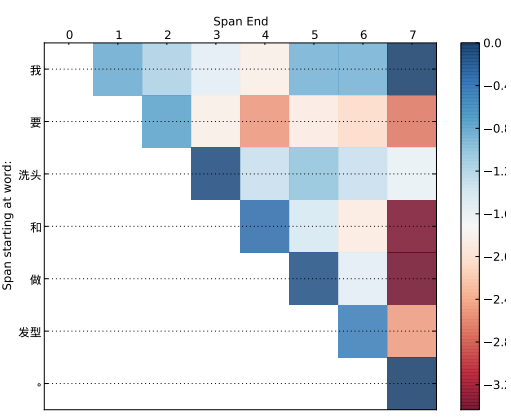

I 'd like a shampoo and style .

(e) Spectral $m=16 \mathrm{RI}$

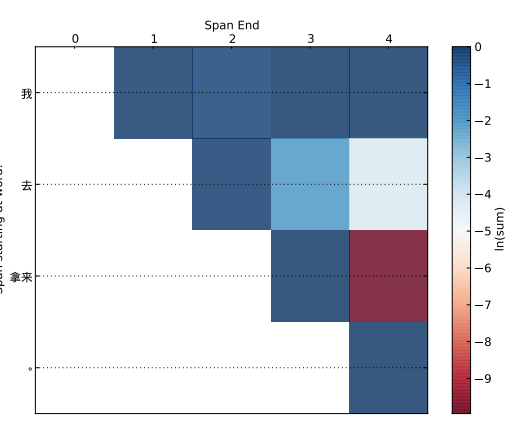

I 'll bring it .

(c) $\mathrm{EM} m=16$

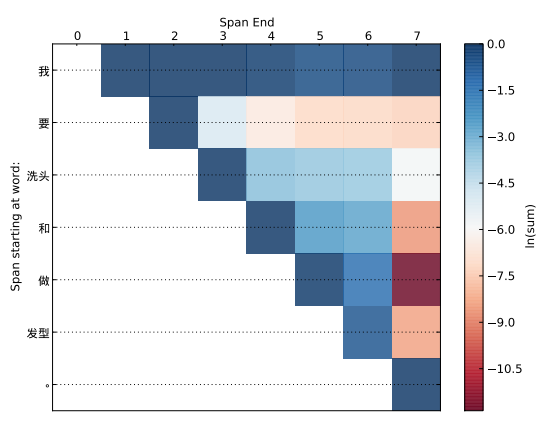

I 'd like a shampoo and style .

(f) $\mathrm{EM} m=16$

Figure 3: A comparison of the CKY charts containing marginal probabilities of non-terminal spans $\mu(\mathbf{X}, i, j)$ for the MLE, spectral $m=16$ with rule indicator features, and EM $m=16$, for the two Chinese sentences. Higher likelihoods are in blue, lower likelihoods in red. The hypotheses produced by each setup are below the heat maps.

els (Mariño et al., 2006; Durrani et al., 2011) seek to model long-distance dependencies and reorderings through $n$-grams. Similarly, Vaswani et al. (2011) use a Markov model in the context of tree-to-string translation, where the parameters are smoothed with absolute discounting (Ney et al., 1994), while in our instance we capture this smoothing effect through low rank or latent states. Feng and Cohn (2013) also utilize a Markov model for MT, but learn the parameters through a more sophisticated estimation technique that makes use of Pitman-Yor hierarchical priors.

Hsu et al. (2009) presented one of the initial efforts at spectral-based parameter estimation (using SVD) of observed moments for latent-variable models, in the case of Hidden Markov models. This idea was extended to L-PCFGs (Cohen et al., 2014), and our approach can be seen as a bilingual or synchronous generalization.

\section{Conclusion}

In this work, we presented an approach to refine synchronous grammars used in MT by inferring the latent categories for the single non- terminal in our grammar rules, and proposed two algorithms to estimate parameters for our latentvariable model. By fixing the synchronous derivations of each parallel sentence in the training data, it is possible to avoid many of the computational issues associated with synchronous grammar induction. Improvements over a minimal grammar baseline and equivalent performance to a hierarchical phrase-based baseline are achieved by the spectral approach. For future work, we will seek to relax this consideration and jointly reason about non-terminal categories and derivation structures.

\section{Acknowledgements}

The authors would like to thank Daniel Gildea for sharing his code to extract minimal derivation trees, Stefan Riezler for useful discussions, Brendan O'Connor for the CKY visualization advice, and the anonymous reviewers for their feedback. This work was supported by a grant from eBay Inc. (Saluja), the U. S. Army Research Laboratory and the U. S. Army Research Office under contract/grant number W911NF-10-1-0533 (Dyer). 


\section{References}

Taylor Berg-Kirkpatrick, Alexandre Bouchard-Côté, John DeNero, and Dan Klein. 2010. Painless unsupervised learning with features. In Proceedings of NAACL.

Phil Blunsom, Trevor Cohn, and Miles Osborne. 2008. Bayesian Synchronous Grammar Induction. In Proceedings of NIPS.

David Chiang. 2007. Hierarchical phrase-based translation. Computational Linguistics, 33(2):201-228, June.

David Chiang. 2012. Hope and Fear for Discriminative Training of Statistical Translation Models. Journal of Machine Learning Research, pages 1159-1187.

Shay B. Cohen and Michael Collins. 2012. Tensor decomposition for fast parsing with latent-variable PCFGs. In Proceedings of NIPS.

Shay B. Cohen, Karl Stratos, Michael Collins, Dean P. Foster, and Lyle Ungar. 2013. Experiments with spectral learning of latent-variable PCFGs. In Proceedings of NAACL.

Shay B. Cohen, Karl Stratos, Michael Collins, Dean P. Foster, and Lyle Ungar. 2014. Spectral learning of latent-variable PCFGs: Algorithms and sample complexity. Journal of Machine Learning Research.

Arthur P. Dempster, Nan M. Laird, and Donald B. Rubin. 1977. Maximum likelihood from incomplete data via the EM algorithm. Journal of the Royal Statistical Society, Series B, 39(1):1-38.

Nadir Durrani, Helmut Schmid, and Alexander Fraser. 2011. A joint sequence translation model with integrated reordering. In Proceedings of ACL.

Chris Dyer, Adam Lopez, Juri Ganitkevitch, Johnathan Weese, Ferhan Ture, Phil Blunsom, Hendra Setiawan, Vladimir Eidelman, and Philip Resnik. 2010. cdec: A decoder, alignment, and learning framework for finite-state and context-free translation models. In Proceedings of ACL.

Yang Feng and Trevor Cohn. 2013. A Markov model of machine translation using non-parametric bayesian inference. In Proceedings of ACL.

Michel Galley, Mark Hopkins, Kevin Knight, and Daniel Marcu. 2004. What's in a translation rule? In Proceedings of HLT-NAACL.

Michel Galley, Jonathan Graehl, Kevin Knight, Daniel Marcu, Steve DeNeefe, Wei Wang, and Ignacio Thayer. 2006. Scalable inference and training of context-rich syntactic translation models. In Proceedings of ACL.

Jonathan Graehl, Kevin Knight, and Jonathan May. 2008. Training tree transducers. Computational Linguistics, 34(3):391-427, September.
Greg Hanneman and Alon Lavie. 2013. Improving syntax-augmented machine translation by coarsening the label set. In Proceedings of NAACL.

Daniel Hsu, Sham M. Kakade, and Tong Zhang. 2009. A Spectral Algorithm for Learning Hidden Markov Models. In Proceedings of COLT.

Liang Huang, Kevin Knight, and Aravind Joshi. 2006. Statistical syntax-directed translation with extended domain of locality. In Proceedings of AMTA.

Zhongqiang Huang, Martin Čmejrek, and Bowen Zhou. 2010. Soft syntactic constraints for hierarchical phrase-based translation using latent syntactic distributions. In Proceedings of EMNLP.

Dan Klein and Christopher D. Manning. 2001. Parsing and hypergraphs. In Proceedings of IWPT.

Philipp Koehn, Franz Josef Och, and Daniel Marcu. 2003. Statistical phrase-based translation. In Proceedings of NAACL.

Abby Levenberg, Chris Dyer, and Phil Blunsom. 2012. A Bayesian model for learning SCFGs with discontiguous rules. In Proceedings of EMNLP-CoNLL.

Percy Liang, Slav Petrov, Michael I. Jordan, and Dan Klein. 2007. The infinite PCFG using hierarchical dirichlet processes. In Proceedings of EMNLP.

José B. Mariño, Rafael E. Banchs, Josep M. Crego, Adrià de Gispert, Patrik Lambert, José A. R. Fonollosa, and Marta R. Costa-jussà. 2006. N-grambased machine translation. Computational Linguistics, 32(4):527-549, December.

Takuya Matsuzaki, Yusuke Miyao, and Jun'ichi Tsujii. 2005. Probabilistic CFG with latent annotations. In Proceedings of ACL.

Markos Mylonakis and Khalil Sima'an. 2011. Learning hierarchical translation structure with linguistic annotations. In Proceedings of ACL.

Hermann Ney, Ute Essen, and Reinhard Kneser. 1994. On Structuring Probabilistic Dependencies in Stochastic Language Modelling. Computer Speech and Language, 8:1-38.

Franz Josef Och. 2003. Minimum error rate training in statistical machine translation. In Proceedings of $A C L$.

Kishore Papineni, Salim Roukos, Todd Ward, and WeiJing Zhu. 2002. BLEU: A method for automatic evaluation of machine translation. In Proceedings of $A C L$.

Michael Paul. 2009. Overview of the IWSLT 2009 evaluation campaign. In Proceedings of IWSLT.

Slav Petrov, Leon Barrett, Romain Thibaux, and Dan Klein. 2006. Learning accurate, compact, and interpretable tree annotation. In Proceedings of ACL. 
Takeaki Uno and Mutsunori Yagiura. 2000. Fast algorithms to enumerate all common intervals of two permutations. Algorithmica, 26(2):290-309.

Ashish Vaswani, Haitao Mi, Liang Huang, and David Chiang. 2011. Rule Markov models for fast tree-tostring translation. In Proceedings of ACL.

Ashish Venugopal, Andreas Zollmann, Noah A. Smith, and Stephan Vogel. 2009. Preference grammars: Softening syntactic constraints to improve statistical machine translation. In Proceedings of NAACL.

Dekai Wu. 1997. Stochastic inversion transduction grammars and bilingual parsing of parallel corpora. Computational Linguistics, 23(3):377-403, September.

Ying Zhang, Stephan Vogel, and Alex Waibel. 2004. Interpreting BLEU/NIST scores: How much improvement do we need to have a better system. In In Proceedings LREC.

Hao Zhang, Daniel Gildea, and David Chiang. 2008. Extracting synchronous grammar rules from wordlevel alignments in linear time. In Proceedings of COLING.

Andreas Zollmann and Ashish Venugopal. 2006. Syntax augmented machine translation via chart parsing. In Proceedings of the Workshop on Statistical Machine Translation, StatMT '06, pages 138-141. Association for Computational Linguistics. 